\title{
Extragalactic Line Surveys
}

\author{
Sergio Martín ${ }^{1}$ \\ ${ }^{1}$ European Southern Observatory, \\ Alonso de Córdova 3107, Vitacura, Casilla 19001, Santiago 19, Chile \\ email: smartin@eso.org
}

\begin{abstract}
Galaxy. Since then, our knowledge on the distribution, kinematics and composition of the molecular material in the extragalactic ISM has built up significantly based not only on the carbon monoxide observations but also in the more than 50 molecular species detected. In particular, line surveys have been proven to be excellent tools to study the chemical composition in the nuclei of galaxies. Such studies have been favored by the increasing instantaneous bandwidth of current $\mathrm{mm}$ and sub-mm facilities. Here I will summarize the highlights of extragalactic molecular spectroscopy, mostly focusing in the results from molecular line surveys published in the last few years as well as the aims of still ongoing projects.
\end{abstract}

Keywords. Astronomical data bases: surveys, Galaxies: abundances, Galaxies: active, Galaxies: ISM, Galaxies: starburst

\section{Introduction: Chemical variations in galactic nuclei}

To date, all of the spectral line surveys towards extragalactic sources have been carried out at low spatial resolution. The large beam sizes in most of these studies just gather the averaged emission over the central few hundred parsecs in the central regions of nearby galaxies. Almost two decades ago, the review paper by Mauersberger \& Henkel (1993) suggested that we could naively expect that the chemical variations in the different molecular cloud complexes, affected by different physical and chemical proccesses, would cancel out when observing the averaged emission over large regions. However, based on a number of previous studies and the more than two dozen of extragalactic molecular detections, they claimed that "surprisingly, the variations in the chemical composition do not cancel out" (Mauersberger \& Henkel 1993). Their comparison of the molecular abundances of just seven species in the starburst galaxy prototypes, M 82 and NGC 253, where only three species were detected in both galaxies, showed that significant differences could be identified. Such abundance differences were attributed to the different heating mechanisms driving the chemistry in their central regions. It became then clear the potential of chemical composition comparative studies to probe the physical processes in the heavily obscured central regions of galaxies. Spectral line surveys, particularly at $\mathrm{mm}$ and submm wavelenghts, became key to understand and stablish the chemical classification and evolution of galactic nuclei.

\section{Targeted vs. Unbiased surveys}

Starburst galaxies have been the evident targets for the first molecular line surveys given the strong molecular emission. Moreover, these galaxies are indeed the most prominent molecular emitters outside the galaxy. The study by Wang et al. (2004) towards 
Table 1. Instantaneous bandwidth in GHz of currently available $\mathrm{mm}$ and submm facilities

\begin{tabular}{lll|lll}
\hline \multicolumn{2}{c}{ Single Dish } & \multicolumn{4}{c}{ Interferometer } \\
\hline MOPRA & $\ldots$ & 8.2 & SMA & $\ldots$ & 8 \\
IRAM $30 \mathrm{~m}$ & $\ldots$ & 8 & CARMA & $\ldots$ & 8 \\
Nobeyama $45 \mathrm{~m}$ & $\ldots$ & 32 & PdBI & $\ldots$ & 4 \\
APEX & $\ldots$ & 8 & ATCA & $\ldots$ & 4 \\
JCMT & $\ldots$ & 1.8 & ALMA & $\ldots$ & 8 \\
\hline
\end{tabular}

the central region of NGC 4945 is likely the first systematic detailed study of the chemical composition in an external galaxy. Using the Swedish-ESO Submillimeter Telescope (SEST), they observed 80 transitions from 19 different species over a frequecy range between 82 and $354 \mathrm{GHz}$. The derived molecular abundances and isotopic ratios measured in NGC 4945 were compared to those available in the literature from M 82 and NGC 253. Though quite complete, this comparison was limited by the selection of the observed molecular species. Line surveys over wide frequency ranges, on the other hand, provide a complete and unbiased description of the chemical composition in galactic nuclei. The first unbiased spectral line survey was carried out with the IRAM $30 \mathrm{~m}$ telescope towards the central region of the starburst galaxy NGC 253 and covered the $2 \mathrm{~mm}$ atmospheric window between 129 and $175 \mathrm{GHz}$ (Martín et al. 2006). This scan resulted in the detection of 111 spectral features from 25 molecular species as well as three hydrogen recombination lines. The follow up towards M 82, the brightest molecular emitter together with NGC 253, surveyed the same $2 \mathrm{~mm}$ window as well as the $1.2 \mathrm{~mm}$ band between 241 and $260 \mathrm{GHz}$ (Aladro et al. Submitted). Although expected from previous observations, the detection rate in M 82 was significantly lower, with 72 spectral features from 18 molecular species. The combination of both line surveys resulted in the most complete comparison between the molecular composition of two extragalactic sources. Indeed, the observed chemical differences are claimed to be directly linked to the different state of evolution of their nuclear starbursts, and therefore to the different leading heating mechanisms affecting the ISM within their central few hundred parsecs. While a number of species, such as $\mathrm{CH}_{3} \mathrm{OH}, \mathrm{HNCO}, \mathrm{NH}_{3}$, $\mathrm{SiO}, \mathrm{NS}, \mathrm{HOCO}$, and $\mathrm{CH}_{2} \mathrm{NH}$, claimed to be formed in dust grains and injected into gas phase via shocks, were found to be enhanced in NGC 253, other molecules, such as $\mathrm{CO}^{+}, \mathrm{HCO}, \mathrm{HOC}^{+}, \mathrm{c}-\mathrm{C}_{3} \mathrm{H}_{2}, \mathrm{CH}_{3} \mathrm{CCH}$, and $\mathrm{NH}_{2} \mathrm{CN}$, mostly formed in the gas phase and likely enhanced by intense UV fields, are significantly more abundant towards M 82. Even though some of these differences were previously known, a number of them (in italics in the list above) were pointed out from the observations covered by the line surveys. In particular, line surveys allow the identification of particularly interesting and contrasted species such as the case of methanimine $\left(\mathrm{NH}_{2} \mathrm{CN}\right)$ which shows rotational temperatures similar to those measured towards the Galactic hot core Sgr B2(M) in both M 82 and NGC 253, but shows an abundance enhancement towards the former.

\section{Receiver Bandwidth Upgrades: Ongoing surveys}

At that time of the first line surveys described in previous section, the available instantaneous bandwidth in both SEST and the IRAM 30m telescopes was $\sim 2 \mathrm{GHz}$. Even with such not inconsiderable bandwidths, spectral scans projects were still significantly 
expensive in terms of telescope time. To illustrate this, the surveys towards M 82 and NGC 253 each required more than one hundred hours of telescope time at the IRAM 30m telescope. However, in the last few years, major upgrades have been performed in all the $\mathrm{mm}$ and submm facilities around the globe. By the end of 2005, the MOPRA telescope commissioned the MOPS digital filterbank spectrometer which provided an $\sim 8 \mathrm{GHz}$ instantaneous bandwidth. Though unique at the time, most single-dish telescopes and interferometers catched up with similarly wide band receivers as shown in Table 1. Just recently, the Nobeyama 45m telescope started commissioning the SAM45 spectrometer, consisting of a copy of the ALMA/ACA correlator that allows a maximum of a $32 \mathrm{GHz}$ bandwidth.

These major telescope upgrades have open the possibility of extending the exploration of wider spectral bands towards larger sample of extragalactic objects given the significantly reduced time required for such spectral scans. Consequently, a number of spectral line surveys have been carried out or are currently ongoing in almost every $\mathrm{mm}$ and submm facility. Table 2 summarizes the observational details of some of the most relevant line surveys carried out at $\mathrm{mm}$ and submm wavelenghts. Here we describe the main aims of theses surveys:

IRAM 30m .- Following the success of the $2 \mathrm{~mm}$ line surveys of NGC 253 and M 82 , a series of deep line survey follow up at $3 \mathrm{~mm}$ are currently ongoing and near completion. This project sample consist of 6 galaxies, including two young starbursts (M 83 and NGC 253), two evolved starbursts (M 82 and $M$ 51), the active galactic nucleus (AGN) prototype NGC 1068 and the ULIRG Arp 220. A total of 36 molecular species are detected in the covered band. This study aims to set the basis of a chemical classification of the extragalactic ISM in the central few hundred parsecs of nearby bright galaxies. The new instrumentation at the IRAM $30 \mathrm{~m}$ telescope reduced the time required for each spectral scan by almost an order of magnitude, down to $\simeq 10$ hours per source.

Nobeyama $45 \mathrm{~m}$.- Three line surveys in the $3 \mathrm{~mm}$ atmospheric band had just been finished at the $45 \mathrm{~m}$ facility making use of the recently upgraded spectrometer (Poster 2.69). With more than a dozen molecular species detected, these surveys will be able to establish the differences between the chemistry in starburst galaxies (NGC 253 and IC 342) and the AGN dominated NGC 1068. Thus the main motivation of this work is to study the chemical fingerprints of the AGN influence on the surrounding molecular gas. The relatively small $\left(18^{\prime \prime}\right)$ beam of the $45 \mathrm{~m}$ telescope is smaller than the starburst ring in NGC 1068, allowing the observation of the chemical composition towards the AGN without contamination from the molecular material in starburst.

APEX 12m .- The spectral scans towards NGC 4945, NGC 253 and Arp 220 with the APEX telescope (Poster 2.83) are exploiting the capabilities of the FLASH receiver at APEX which processes an $8 \mathrm{GHz}$ instantaneous bandwidth. These surveys are exploring the higher energy transitions in the $0.9 \mathrm{~mm}$ atmospheric window. A total of 16 molecular species are detected at the highest frequencies ever surveyed outside the Galaxy. Additionally, NGC 253 has been surveyed in the $1.3 \mathrm{~mm}$ atmospheric window from 185 to $275 \mathrm{GHz}$ (Poster 2.83). These APEX scans complete the spectral coverage towards NGC 253 over a total band of $274 \mathrm{GHz}$ between 3.5 and $0.8 \mathrm{~mm}$ (see Table 2).

$S M A$.- The Submillimeter Array was used to carry out the first interferometric extragalactic unbiased survey (see Section 6). The large width of the emission lines in Arp 220 together with the strong line confusion in this object, made it necessary to use the stable baselines achieved with the interferometer. A total of 70 spectral features from 15 molecular species and 6 isotopologues were identified. The survey was partially confusion limited, with an average of 1.8 lines per GHz. This spectral scan was the first 
Table 2. Extragalactic spectral scans at $\mathrm{mm}$ and submm wavelenghts

\begin{tabular}{|c|c|c|c|c|c|}
\hline Source & $\begin{array}{l}\text { Frequency } \\
\text { Range }(\mathrm{GHz})\end{array}$ & Telescope & $\begin{array}{l}\text { Resolution } \\
\left(\mathrm{km} \mathrm{s}^{-1}\right)\end{array}$ & $\begin{array}{l}\mathrm{rms} \\
(\mathrm{mK})\end{array}$ & Reference \\
\hline \multirow[t]{5}{*}{ NGC 253} & $86-116$ & IRAM 30m & 12 & 2 & Aladro et al. In prep. \\
\hline & $85-116$ & Nobeyama $45 \mathrm{~m}$ & 20 & $2-13$ & Nakajima et al. In prep. \\
\hline & $129-175$ & IRAM 30m & 8 & $2-6$ & Martín et al. (2006) \\
\hline & $185-275$ & APEX $12 \mathrm{~m}$ & 12 & 2 & Requena-Torres et al. In prep. \\
\hline & $280-360$ & APEX 12m & 20 & 2 & Requena-Torres et al. In prep. \\
\hline \multirow[t]{3}{*}{ M 82} & $86-116$ & IRAM 30m & 12 & 2 & Aladro et al. In prep. \\
\hline & $130-175$ & IRAM 30m & 8 & $2-5$ & Aladro et al. Submitted \\
\hline & $241-260$ & IRAM 30m & 5 & $4-6$ & Aladro et al. Submitted \\
\hline \multirow[t]{2}{*}{ NGC 1068} & $86-116$ & IRAM $30 \mathrm{~m}$ & 12 & 2 & Aladro et al. In prep. \\
\hline & $85-116$ & Nobeyama $45 \mathrm{~m}$ & 20 & $1-4$ & Nakajima et al. In prep. \\
\hline M 83 & $86-116$ & IRAM 30m & 12 & 2 & Aladro et al. In prep. \\
\hline M 51 & $86-116$ & IRAM 30m & 12 & 2 & Aladro et al. In prep. \\
\hline \multirow[t]{3}{*}{ Arp 220} & $86-116$ & IRAM 30m & 12 & 2 & Aladro et al. In prep. \\
\hline & $202-242$ & SMA & 250 & $4^{1}$ & Martín et al. (2011) \\
\hline & $280-360$ & APEX 12m & 20 & 2 & Requena-Torres et al. In prep. \\
\hline IC 342 & $85-116$ & Nobeyama $45 \mathrm{~m}$ & 20 & $1-2$ & Nakajima et al. In prep. \\
\hline NGC 4945 & $280-360$ & APEX 12m & 20 & 2 & Requena-Torres et al. In prep. \\
\hline PKS 1830-211 & $30-50$ & ATCA & $6-10$ & $\ldots$ & Muller et al. (2011) \\
\hline
\end{tabular}

Notes:

${ }^{1} \mathrm{rms}$ in $\mathrm{mJy}_{\text {beam }}{ }^{-1}$ units

carried out towards a ULIRG at a distance of $70 \mathrm{Mpc}$, an order of magnitude farther than nearby starburst such as NGC 253 and M 82. Some of the highlights of this study are summarized in Section 6.

\section{Going wider: high redshift wide band receivers}

In the last few years, a number of extremely wide band receivers have been developed to measure the redshift of distant heavily obscured objects. These receiver cover wide frequency bands at a low spectral resolution. Even if this spectral resolution is similar or even wider than the tipical linewidth of local galaxies, it is still possible to study the brightest molecular transitions in relatively large samples of galaxies at a relatively low observing time expense. In this section we summarize some of the latest publications making use of these wide band instruments.

Redshift Search Receiver (RSR) at FCRAO 14m .- The RSR, design as a facility instrument for the $50 \mathrm{~m}$ LMT, is a dual polarization dual beam instrument with 4 broadband receivers that covers the $37 \mathrm{GHz}$ accross the $3 \mathrm{~mm}$ atmospheric window, between 74 and $111 \mathrm{GHz}$. The spectral resolution of $\sim 100 \mathrm{~km} \mathrm{~s}^{-1}$ provided is barely enough to resolve the typical linewidth of $\sim 200 \mathrm{~km} \mathrm{~s}^{-1}$ in the central region of local galaxies. This spectrometer was used to survey the $3 \mathrm{~mm}$ atmospheric band towards a sample of 10 galaxies with different types of nuclear activity (see Table 1 in Snell et al. 2011). A total of 33 transition of 13 molecular species were detected. The low number of spectral features as compared with similar scans with the IRAM $30 \mathrm{~m}$ or Nobeyama $45 \mathrm{~m}$ is a consequence of the line smoothing due to the low spectra resolution. Still line ratios between the brighter spectral features could be extracted from this survey. For example the $\mathrm{HCO}^{+} / \mathrm{HCN}$ ratio 
was observed to be enhanced in AGN dominated galaxies when compared to starburst (SB) dominated galaxies (Snell et al. 2011). This result, in disagreement with the result from Krips et al. (2008), where the ratio $\mathrm{HCO}^{+} / \mathrm{HCN}$ is observed to be enhanced in $\mathrm{SB}$ galaxies, shows the difficulty of establishing activity templates in extragalactic sources. It is indeed difficult to disantangle the contribution from AGN and SB at the low resolution provided by single dish observations. As such, the highest $\mathrm{HCO}^{+} / \mathrm{HCN}$ ratios from the surveys from Snell et al. (2011) were derived from the LIRGs NGC 3690 and NGC 6240 where, even if known to host an active nucleus, the contribution from the star formation cannot be excluded.

Z-Spec at CSO 10.4m - Z-Spec is a millimeter wave grating spectrometer dispersing the light to an array of 160 bolometers. Though covering an enormous bandwidth of almost $120 \mathrm{GHz}$ from 190 to $307 \mathrm{GHz}$, the spectral resolution is very low. Velocity resolution ranges from $700 \mathrm{~km} \mathrm{~s}^{-1}$ at the lower frequencies to $1200 \mathrm{~km} \mathrm{~s}^{-1}$ at the higher end. This resolution is significantly coarser than the observed molecular linewidth even in distant galaxies. Still, this receiver has been used to survey large frequency bands in local galaxies such as the 3 positions observed towards the starburst galaxy M 82 (Naylor et al. 2010). With an integration of just 1 hour per position, 10 molecular species were detected within the band. Even though the resolution was heavily limited, it is possible to simultaneous observe three transitions of CS and therefore to constrain and compare the physical properties of the molecular gas in the three observed positions. Similarly, Kamenetzky et al. (2011) observed the central region of NGC 1068 with Z-spec, where they identified 12 molecular species.

Though not intended as a molecular line survey, the observations with Z-Spec towards teh Cloverleaf galaxy at $\mathrm{z} \sim 2.56$ covered the rest frame wavelenghts between 272 and $444 \mu \mathrm{m}$ (Bradford et al. 2009). At these wavelenghts, four transition of CO (from $J=6-5$ up to $9-8)$ were clearly detected and [CI] at $370 \mu \mathrm{m}$ blended to the CO $7-6$ transition. Additionally, tentative detection of $\mathrm{H}_{2} \mathrm{O}$ in emission and $\mathrm{CH}^{+}$and $\mathrm{LiH}^{+}$in absorption were reported. These $\mathrm{CO}$ simultaneous measurements, together with previous lower- $J$ detections with the IRAM PdBI, allow the detailed study of the CO spectral line energy distribution (SLED) in the cloverleaf (Figure 2 in Bradford et al. 2009). The studies at high-z with broad band spectrometers at ground based telescopes are similar to those carried out with Herschel space observatory toward nearby galaxies. For example, the observations with the SPIRE spectrometer covering the frequencies from 467 to $989 \mathrm{GHz}$ measured the CO emission from $J=5-4$ to $13-12$ towards the ULIRG Mrk 231 (van der Werf et al. 2010). The resulting CO SLED has been modelled with a combination of dense gas exposed to a strong UV radiation and a significant X-ray contribution from the supermassive black hole to explain the line luminosity of the higher transitions. Similar SLED were modelled towards M 82 based on both SPIRE and HIFI spectrometers on board Herschel (Panuzzo et al. 2010; Loenen et al. 2010). The SPIRE spectrum towards Mrk 231 (van der Werf et al. 2010) also resulted in the detection rotational lines of $\mathrm{H}_{2} \mathrm{O}, \mathrm{OH}^{+}, \mathrm{H}_{2} \mathrm{O}^{+}, \mathrm{CH}^{+}$, and $\mathrm{HF}$, which shows the potential of molecular studies in the submillimeter to infrared wavelenghts.

\section{Absorption systems}

Among the surveys compiled in this review, those towards absorption systems deserve an special mention due to its enormous potential to carry out molecular studies at intermediate redshift. Such is the case of the spectral scan carried out with ATCA towards 
Table 3. Census of extragalactic molecular species and isotopologues detected

\begin{tabular}{|c|c|c|c|c|c|}
\hline 2 atoms & 3 atoms & 4 atoms & 5 atoms & 6 atoms & 7 atoms \\
\hline $\mathrm{OH}$ & $\mathrm{H}_{2} \mathrm{O}, \mathrm{H}_{2}^{18} \mathrm{O}$ & $\mathrm{H}_{2} \mathrm{CO}$ & $\mathrm{c}-\mathrm{C}_{3} \mathrm{H}_{2}$ & $\mathrm{CH}_{3} \mathrm{OH},{ }^{13} \mathrm{CH}_{3} \mathrm{OH}$ & $\mathrm{CH}_{3} \mathrm{C}_{2} \mathrm{H}$ \\
\hline $\mathrm{CO}\left\{\begin{array}{l}13 C_{O} O \\
C^{18} O \\
C^{17} O\end{array}\right.$ & $\mathrm{HCN}\left\{\begin{array}{l}H^{13} C N \\
H C^{15} N \\
D C N\end{array}\right.$ & $\mathrm{NH}_{3}$ & $\mathrm{HC}_{3} \mathrm{~N}\left\{\begin{array}{l}H^{13} C C C N \\
H C^{13} C C N \\
H C C^{13} C N\end{array}\right.$ & $\mathrm{CH}_{3} \mathrm{CN}$ & $\mathrm{CH}_{3} \mathrm{NH}_{2}$ \\
\hline $\mathrm{H}_{2}, H D$ & $\mathrm{HCO}^{+}\left\{\begin{array}{l}H^{13} \mathrm{CO}^{+} \\
\mathrm{HC}^{18} \mathrm{O}^{+} \\
D C O^{+}\end{array}\right.$ & $\mathrm{HNCO}$ & $\mathrm{CH}_{2} \mathrm{NH}$ & & $\mathrm{CH}_{3} \mathrm{CHO}$ \\
\hline $\mathrm{CH}$ & $\mathrm{C}_{2} \mathrm{H}$ & $\mathrm{H}_{2} \mathrm{CS}$ & $\mathrm{NH}_{2} \mathrm{CN}$ & & \\
\hline $\mathrm{CS}\left\{\begin{array}{l}13 C_{S} \\
C^{34} S \\
C^{33} S\end{array}\right.$ & 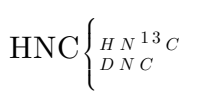 & $\mathrm{HOCO}^{+}$ & $\mathrm{CH}_{2} \mathrm{CO}$ & & \\
\hline $\mathrm{CH}^{+}$ & $\mathrm{N}_{2} \mathrm{H}^{+}, N_{2} D^{+}$ & $\mathrm{C}_{3} \mathrm{H}$ & $1-\mathrm{C}_{3} \mathrm{H}_{2}$ & & \\
\hline $\mathrm{CN}$ & OCS & $\mathrm{H}_{3} \mathrm{O}^{+}$ & $\mathrm{H}_{2} \mathrm{CCN}$ & & \\
\hline $\mathrm{SO},{ }^{34} \mathrm{SO}$ & $\mathrm{HCO}$ & & $\mathrm{H}_{2} \mathrm{CCO}$ & & \\
\hline $\mathrm{SiO},{ }^{29} \mathrm{SiO}$ & $\mathrm{H}_{2} \mathrm{~S}$ & & $\mathrm{C}_{4} \mathrm{H}$ & & \\
\hline $\mathrm{CO}^{+}$ & $\mathrm{SO}_{2}$ & & & & \\
\hline $\mathrm{NO}$ & $\mathrm{HOC}^{+}$ & & & & \\
\hline NS & $\mathrm{C}_{2} \mathrm{~S}$ & & & & \\
\hline $\mathrm{LiH}$ & $\mathrm{H}_{3}^{+}$ & & & & \\
\hline $\mathrm{CH}$ & $\mathrm{H}_{2} \mathrm{O}^{+}$ & & & & \\
\hline $\mathrm{NH}$ & $1-\mathrm{C}_{3} \mathrm{H}$ & & & & \\
\hline $\mathrm{OH}^{+}$ & & & & & \\
\hline $\mathrm{HF}$ & & & & & \\
\hline $\mathrm{SO}^{+}$ & & & & & \\
\hline
\end{tabular}

Notes: Updated Table from Martín et al. (2011) with the new detections in the absorption system PKS 1830-211 by Muller et al. (2011).

the absorption system PKS 1830-211 (Muller et al. 2011). The survey (see Invited talk in this volume by Sebastien Muller for an extensive description of the results) covered the frequency range from 30 to $50 \mathrm{GHz}$, equivalent to the $5-3 \mathrm{~mm}$ rest frame between 57 and $111 \mathrm{GHz}$ at the redshift of $\mathrm{z}=0.89$. As a result of the spectral scan 28 molecular species and 8 isotopologues were identified. This study turns PKS 1830-211 into one of the extragalactic sources with the largest number of molecular detections. The accurate fractional abundances derived from these observations allows complete comparison with Galactic sources (see Fig. 8 in Muller et al. 2011) which showed the similar molecular composition between this absorption system and that found in typical Galactic diffuse and translucent clouds.

\section{The chemistry in the Arp 220 ULIRG}

At a redshift of $z=0.018$, Arp 220 is the nearest ULIRG. With a star formation rate of SFR $300 M_{\odot} y r^{-1}$, this galaxy could be considered as a scaled down version of the galaxies observed at high redshift, with SFR up to an order of magnitude higher than that in Arp 220. The $1.3 \mathrm{~mm}$ line survey carried out with the SMA (Sect. 3) aimed to establish the differences and similarities between the molecular composition in ULIRGs and that found in local nearby starburst galaxies. Moreover, it aimed to study whether the nuclear 
power source, AGN and/or SB, had an imprint in the chemical composition. Previous to this spectral scan, a number of molecular studies had been carried out towards this source. The paper by Greve et al. (2009) presented a complete compilation of all single dish observations of $\mathrm{CO}, \mathrm{HCN}, \mathrm{HCO}^{+}, \mathrm{HNC}$ and $\mathrm{HNCO}$, as well as $\mathrm{CO}$ isotopologues in Arp 220. A detailed study of the molecular content was also carried out in absorption with the Infrared Space Observatory (ISO) between 25 to $1300 \mu \mathrm{m}$ (González-Alfonso et al. 2004).

The overall chemical composition derived from the $1.3 \mathrm{~mm}$ band emission lines resemble that in starbursts such as NGC 253. However, the emission from vibrationally excited transitions of $\mathrm{HC}_{3} \mathrm{~N}$ and $\mathrm{CH}_{3} \mathrm{CN}$, never detected in local starbursts, was detected towards Arp 220 (Martín et al. 2011). Vibrational temperatures derived from these transitions are in the range of $T_{\mathrm{vib}}=300-500 \mathrm{~K}$. Within our Galaxy, vibrationally excited emission of $\mathrm{HC}_{3} \mathrm{~N}$ is observed to be tracing the hottest gas around star forming cores (de Vicente et al. 1997, 2000). In the extragalactic ISM, vibrational emission of $\mathrm{HCN}$ and $\mathrm{HC}_{3} \mathrm{~N}$ have also been recently detected towards the also heavily obscured nucleus of the LIRG NGC 4418 (Costagliola \& Aalto 2010; Sakamoto et al. 2010), as well as $v_{2}=1$-type absorption lines of HCN towards Arp 220 (Salter et al. 2008) and mid-infrared vibration-rotation bands of $\mathrm{C}_{2} \mathrm{H}_{2}, \mathrm{HCN}$, and $\mathrm{CO}_{2}$ in a sample of (U)LIRGs (Lahuis et al. 2007). The high vibrational temperatures derived from these detections is suggested to be the consequence of the IR-pumping (Sakamoto et al. 2010). Though a hot component associated to a buried AGN cannot be excluded (Costagliola \& Aalto 2010), the detected vibrationally excited emission has been claimed to be most likely tracing the warmest gas in the extremely compact star forming regions within these heavily obscured environments (Martín et al. 2011). This scenario is supported by the detection of the ${ }^{18} \mathrm{O}$ isotopologue of water in the Arp $2201.3 \mathrm{~mm}$ line survey. The water abundance estimated from the $\mathrm{H}_{2}^{18} \mathrm{O} / \mathrm{C}^{18} \mathrm{O}$ ratio of $\sim 2 \times 10^{5}$ is similar to that measured in Galactic hot cores (Gensheimer et al. 1996; Cernicharo et al. 2006). Such water detection should imply an enormous star formation concentration of the order of a few $10^{6} \mathrm{Sgr}$ B2(N)-like hot cores in a $\sim 700$ pc region (Martín et al. 2011).

\section{Molecular Census Evolution}

To date, a total of 54 molecular species and 23 isotopologues have been claimed to be detected in the extragalactic interestellar medium. Table 3 shows a similar table to that presented in Martín et al. (2011) which became outdated by the recent detection of $\mathrm{SO}^{+}$, l- $\mathrm{C}_{3} \mathrm{H}, \mathrm{l}_{-} \mathrm{C}_{3} \mathrm{H}_{2}, \mathrm{H}_{2} \mathrm{CCN}, \mathrm{H}_{2} \mathrm{CCO}, \mathrm{C}_{4} \mathrm{H}, \mathrm{CH}_{3} \mathrm{NH}$, and $\mathrm{CH}_{3} \mathrm{CHO}$ in the spectral scan towards the absorption system PKS 1830-211 (see Sect. 5). It is definitely worth having a look on how the census of extragalactic molecular detections has evolved as a function of time. Fig. 1 shows the evolution with time of the cumulative number of molecular species detected in the extragalactic ISM. Since the first molecular detection 40 years ago $(\mathrm{OH}$ in absorption, Weliachew 1971) there has been a steady increase in the number of molecular detections. However, we can note two periods in which the rate of detections has been quicken. First, mostly in the period between 1985 and 1990, the increase was due to the availability of sensitive radio facilities, and in particular to the large collecting area of the IRAM 30m telescope. Only in the last few years, from 2003 up until now, spectral line scans have played the dominant role in terms of detection rate. The advent of ALMA, with a significant increase in the collecting area of about an order of magnitud compared to the largest current facilities, will be the next step in sensitivity. In the next decade such increase in sensitivity will allow us to match the number of 


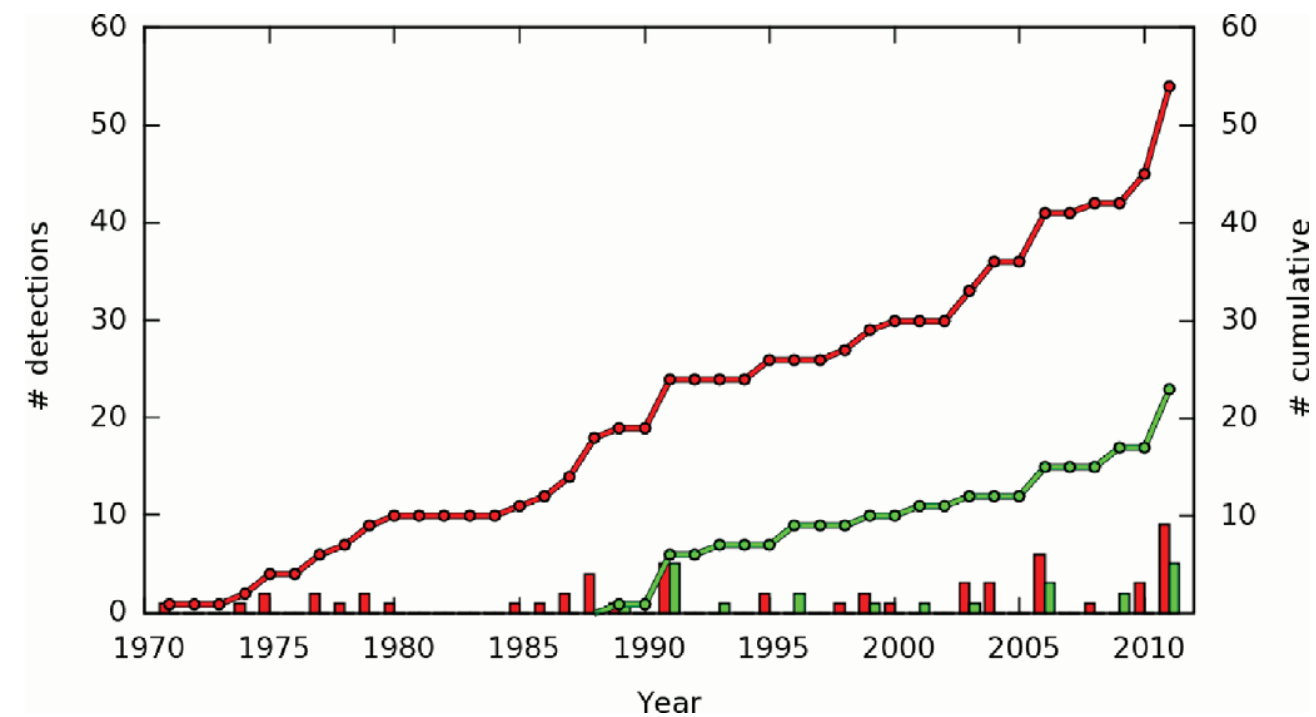

Figure 1. Histogram shows the number of extragalactic molecular detections per year for both the main (in red) and rarer (in green) isotopologues. The connected circles shows the cumulative number of detections.

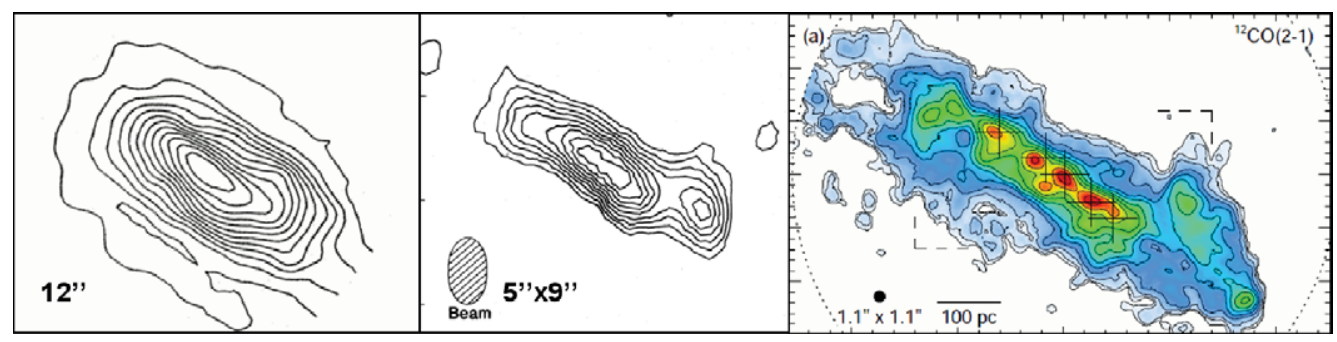

Figure 2. CO integrated emission maps of the starburst galaxy NGC 253 at different spatial resolutions. (left) CO $1-0$ map at $12^{\prime \prime}$ resolution by Mauersberger et al. (1996) with the IRAM $30 \mathrm{~m}$ telescope. (center) CO $2-1$ map at $5^{\prime \prime} \times 9^{\prime \prime}$ resolution by Canzian et al. (1988) with the OVRO interferometer. (right) CO $1-0$ map at $1.1^{\prime \prime}$ resolution by Sakamoto et al. (2011).

extragalactic molecular detections to the number of detected species in the Galactic ISM.

\section{High Resolution Line Surveys}

Even though sensitivity is an key factor for spectral scans, the next step in molecular line surveys does not only applies to sensitivity but also to resolution. Fig. 2 shows the increase in both richness and complexity of the molecular structure in the central few hundred parsecs of NGC 253 as the resolution improves from single dish resolution to high resolution interferometric observations. The high resolution maps by Sakamoto et al. (2011) reached a similar spatial resolution to that achieved in large scale surveys towards 
the Galactic center with small telescopes $(\varnothing=60 \mathrm{~cm}$, Sawada et al. 2001$)$. The imaging capabilities of instruments like ALMA, together with the large bandwidth of the receivers will allow to carry out deep $3 \mathrm{D}$ spectral scans. It will be possible to study the chemical composition variations accross the center of nearby galaxies at a similar resolution to that currently achieved by large single dish telescopes towards our very Galactic center. Thus, we expect line surveys will move a step further from studying the averaged molecular composition over the central few hundred parsecs of nearby galaxies to spatially resolve the chemical composition of individual molecular complexes.

\section{References}

Bradford, C. M., Aguirre, J. E., Aikin, R., et al. 2009, ApJ, 705, 112

Canzian, B., Mundy, L. G., \& Scoville, N. Z. 1988, ApJ, 333, 157

Cernicharo, J., Goicoechea, J. R., Pardo, J. R., \& Asensio-Ramos, A. 2006, ApJ, 642, 940

Costagliola, F. \& Aalto, S. 2010, A\&A, 515, A71

de Vicente, P., Martín-Pintado, J., Neri, R., \& Colom, P. 2000, A\& $A, 361,1058$

de Vicente, P., Martin-Pintado, J., \& Wilson, T. L. 1997, A\& A, 320, 957

Gensheimer, P. D., Mauersberger, R., \& Wilson, T. L. 1996, A\&\&A, 314, 281

González-Alfonso, E., Smith, H. A., Fischer, J., \& Cernicharo, J. 2004, ApJ, 613, 247

Greve, T. R., Papadopoulos, P. P., Gao, Y., \& Radford, S. J. E. 2009, ApJ, 692, 1432

Kamenetzky, J., Glenn, J., Maloney, P. R., et al. 2011, ApJ, 731, 83

Krips, M., Neri, R., García-Burillo, S., et al. 2008, ApJ, 677, 262

Lahuis, F., Spoon, H. W. W., Tielens, A. G. G. M., et al. 2007, ApJ, 659, 296

Loenen, A. F., van der Werf, P. P., Güsten, R., et al. 2010, A\&̊A, 521, L2

Martín, S., Krips, M., Martín-Pintado, J., et al. 2011, A\&A, 527, A36

Martín, S., Mauersberger, R., Martín-Pintado, J., Henkel, C., \& García-Burillo, S. 2006, ApJS, 164,450

Mauersberger, R. \& Henkel, C. 1993, in Reviews in Modern Astronomy, Vol. 6, Reviews in Modern Astronomy, ed. G. Klare, 69-102

Mauersberger, R., Henkel, C., Wielebinski, R., Wiklind, T., \& Reuter, H. 1996, A\&AA, 305, 421

Muller, S., Beelen, A., Guélin, M., et al. 2011, ArXiv e-prints

Naylor, B. J., Bradford, C. M., Aguirre, J. E., et al. 2010, ApJ, 722, 668

Panuzzo, P., Rangwala, N., Rykala, A., et al. 2010, A\& A, 518, L37

Sakamoto, K., Aalto, S., Evans, A. S., Wiedner, M. C., \& Wilner, D. J. 2010, ApJ, 725, L228

Sakamoto, K., Mao, R.-Q., Matsushita, S., et al. 2011, ArXiv e-prints

Salter, C. J., Ghosh, T., Catinella, B., et al. 2008, AJ, 136, 389

Sawada, T., Hasegawa, T., Handa, T., et al. 2001, ApJS, 136, 189

Snell, R. L., Narayanan, G., Yun, M. S., et al. 2011, AJ, 141, 38

van der Werf, P. P., Isaak, K. G., Meijerink, R., et al. 2010, A\&A, 518, L42

Wang, M., Henkel, C., Chin, Y., et al. 2004, A\& A, 422, 883

Weliachew, L. 1971, ApJ, 167, L47

\section{Discussion}

CASElli: You talk about "hot core" species. Did you try to compare abundance ratios in Arp 220 with typical "hot core" abundances, to test if we are indeed observing $\sim 10^{6}$ Sgr B2 hot cores? 
MARTIN: Such comparison was performed with the abundances measured towards the starburst galaxy NGC 253, but not yet with Arp 220. However, Arp 220 and NGC 253 show significantly similar abundances but for the vibrational emission observed towards Arp 220. This difference points out towards a "hot core" origin but a closer comparison with Galactic templates is yet to be performed. 